\title{
Vectors, Cyclic Submodules, and Projective Spaces Linked with Ternions
}

\author{
Hans Havlicek Metod Saniga
}

October 23, 2008

\begin{abstract}
Given a ring of ternions $R$, i. e., a ring isomorphic to that of upper triangular $2 \times 2$ matrices with entries from an arbitrary commutative field $F$, a complete classification is performed of the vectors from the free left $R$-module $R^{n+1}, n \geq 1$, and of the cyclic submodules generated by these vectors. The vectors fall into $5+|F|$ and the submodules into 6 distinct orbits under the action of the general linear group $\mathrm{GL}_{n+1}(R)$.

Particular attention is paid to free cyclic submodules generated by $n o n$-unimodular vectors, as these are linked with the lines of $\operatorname{PG}(n, F)$, the $n$-dimensional projective space over $F$. In the finite case, $F=\mathrm{GF}(q)$, explicit formulas are derived for both the total number of non-unimodular free cyclic submodules and the number of such submodules passing through a given vector. These formulas yield a combinatorial approach to the lines and points of $\operatorname{PG}(n, q), n \geq 2$, in terms of vectors and non-unimodular free cyclic submodules of $R^{n+1}$.
\end{abstract}

Mathematics Subject Classification (2000): 51C05, 51Exx, 16D40.

Key words: Rings of ternions, (non-unimodular free) cyclic submodules, projective lattice geometry over ternions.

\section{Introduction}

Projective spaces over rings (see [10] for the standard terminology, notation and the necessary background information), and projective lines in particular (see [1]), have recently become the subject of considerable interest due to rather unexpected recognition of their relevance for the field of quantum physics in general and quantum information theory in particular; we refer to [5], [6], and the references therein. Being motivated by these intriguing applications, we have had - in the framework of a broader international collaboration - a detailed look at the structure of a variety of finite projective ring lines and planes (see, e. $\mathrm{g}$. [9]) and came across some interesting aspects (see, among others, [7]) which, to the best of our knowledge, have not yet been the subject of a systematic mathematical treatment. These aspects mostly relate to the properties of free cyclic submodules generated by vectors of a free $R$-module of a given unital ring $R$, and can be summarised into the following open problems: how the interrelation between different free cyclic submodules over a particular ring is encoded in the structure of the ideals of the ring; what kind of finite rings feature "outliers", i.e., vectors not belonging to any free cyclic submodule generated 
by unimodular vectors; what the conditions are for a non-unimodular vector to generate a free cyclic submodule; and, finally, how the substructure generated by such non-unimodular free cyclic submodules relates to the parent ring geometry. These questions lead to projective lattice geometry in the sense of [2]. In order to partially answer some of them, we have already examined the case of the smallest ring of ternions [8] - this being, remarkably, the lowest order ring where one not only finds "outliers", but also free cyclic submodules generated by (some of) them. In the present paper we extend and generalise the findings of [8] to an arbitrary ring of ternions, with finite cases handled in somewhat more detail.

\section{Ternions}

Let $F$ be a (commutative) field. We denote by $R$ the ring of ternions, i. e., upper triangular $2 \times 2$ matrices over $F$, with the usual addition and multiplication for matrices. The ring $R$ is non-commutative, with $I$ (the $2 \times 2$ identity matrix over $F$ ) being its multiplicative identity and 0 (the $2 \times 2$ zero matrix over $F$ ) the additive one. ${ }^{1}$ The ring $R$ has precisely two (two-sided) ideals other than 0 and $R$, namely the sets

$$
\mathcal{I}_{1}:=\left\{\left(\begin{array}{ll}
0 & y \\
0 & z
\end{array}\right) \mid y, z \in F\right\} \quad \text { and } \quad \mathcal{I}_{2}:=\left\{\left(\begin{array}{ll}
x & y \\
0 & 0
\end{array}\right) \mid x, y \in F\right\} .
$$

Furthermore, all sets

$$
\mathcal{I}_{1}(b: c):=\left\{\left(\begin{array}{ll}
0 & z b \\
0 & z c
\end{array}\right) \mid z \in F\right\} \text { with }(0,0) \neq(b, c) \in F^{2}
$$

are proper right ideals of $R$. In fact, $\mathcal{I}_{1}(b: c)$ depends only on the ratio $b: c$. Note that the ratio $1: 0$ is also allowed here. Similarly, all sets

$$
\mathcal{I}_{2}(a: b):=\left\{\left(\begin{array}{cc}
x a & x b \\
0 & 0
\end{array}\right) \mid x \in F\right\} \text { with }(0,0) \neq(a, b) \in F^{2}
$$

are proper left ideals of $R$. It is an easy exercise to show that there are no other proper one-sided ideals in $R$ apart from the ones given by (2) and (3). Recall that the Jacobson radical of $R$ (denoted by $\operatorname{rad} R$ ) equals the intersection of all maximal left (or right) ideals. So

$$
\operatorname{rad} R=\mathcal{I}_{1}(1: 0)=\mathcal{I}_{2}(0: 1)
$$

Note that a ternion is invertible (a unit) if, and only if, its diagonal entries are non-zero. The set of invertible ternions will be written as $R^{*}$. It is a group under multiplication.

\section{Classifying vectors and cyclic submodules}

We consider now the free left $R$-module $R^{n+1}$ for some integer $n \geq 1$. (The assumption $n \geq 1$ is needed, for example, to guarantee that all six cases which

\footnotetext{
${ }^{1}$ In what follows, the symbol "0" stands, by abuse of notation, for both the zero-element of the field $F$ and the zero matrix of $R$, the difference being always fairly obvious from the context.
} 
appear in the proof of Lemma 1 below actually occur. We refrain from discussing the trivial cases $n=-1$ and $n=0$ throughout the paper.) We use boldface letters for vectors and matrices with entries from $R$. Any $s \times t$ matrix $\left(A_{i j}\right)$ over $R$ can be considered as a $2 s \times 2 t$ matrix over $F$ which is partitioned in $2 \times 2$ blocks formed by the upper triangular matrices $A_{i j}$, and vice versa. Multiplication of matrices over $R$ is equivalent to multiplication of matrices over $F$ under this oneone correspondence. Thus, in particular, it is easy to check whether a square matrix over $R$ is invertible by calculating the determinant of the associated matrix over $F$.

Our first aim is to classify the (row) vectors of the free left module $R^{n+1}$ up to the natural action of the general linear group $\mathrm{GL}_{n+1}(R)$. Given a vector $\boldsymbol{X}=\left(X_{0}, X_{1}, \ldots, X_{n}\right) \in R^{n+1}$ let $\mathcal{I}_{\boldsymbol{X}}$ denote the right ideal of $R$ which is generated by $X_{0}, X_{1}, \ldots, X_{n}$.

Lemma 1. Two vectors $\boldsymbol{X}, \boldsymbol{Y} \in R^{n+1}$ are in the same $\mathrm{GL}_{n+1}(R)$-orbit if, and only if, the right ideals $\mathcal{I}_{\boldsymbol{X}}$ and $\mathcal{I}_{\boldsymbol{Y}}$ coincide.

Proof. If a vector $\boldsymbol{X} \in R^{n+1}$ is multiplied by a matrix $\boldsymbol{A} \in \mathrm{GL}_{n+1}(R)$, then the coordinates of $\boldsymbol{Y}:=\boldsymbol{X} \cdot \boldsymbol{A}$ belong to $\mathcal{I}_{\boldsymbol{X}}$. By virtue of the inverse matrix $\boldsymbol{A}^{-1}$, we see that actually $\mathcal{I}_{\boldsymbol{X}}=\mathcal{I}_{\boldsymbol{Y}}$.

In order to show the converse, we establish that the orbit of any vector $\boldsymbol{X}=\left(X_{0}, X_{1}, \ldots, X_{n}\right)$ contains a distinguished vector which depends only on the right ideal $\mathcal{I}_{\boldsymbol{X}}$. In our discussion below we make use of two obvious facts. Firstly, we may permute the coordinates of a vector in an arbitrary way by multiplying it with a permutation matrix. Secondly, if $\boldsymbol{X}$ is a non-zero vector with $X_{0} \neq 0$, say, then one of the entries of the ternion $X_{0}$ is a scalar $w \neq 0$. Multiplying $\boldsymbol{X}$ by the invertible matrix $\boldsymbol{A}=\operatorname{diag}\left(w^{-1} I, I, \ldots, I\right)$ gives a vector $\boldsymbol{X}^{\prime}:=\boldsymbol{X} \cdot \boldsymbol{A}$ such that the entry of the ternion $X_{0}^{\prime}$ at the same position equals $1 \in F$. So, without loss of generality, we may assume $w=1$ from the very beginning.

Case 1: $\mathcal{I}_{\boldsymbol{X}}=0$, so that $\boldsymbol{X}=(0,0, \ldots, 0)$ is already the distinguished vector.

Case 2: $\mathcal{I}_{\boldsymbol{X}}=\operatorname{rad} R$. Thus $\boldsymbol{X}$ has the form $\left(X_{0}, X_{1}, \ldots, X_{n}\right)$ with $X_{i}=$ $\left(\begin{array}{cc}0 & y_{i} \\ 0 & 0\end{array}\right)$ and $y_{0}=1$, say. Multiplying $\boldsymbol{X}$ by the invertible matrix

$$
\left(\begin{array}{cccc}
I & -y_{1} I & \ldots & -y_{n} I \\
0 & I & \ldots & 0 \\
\ldots \ldots & \ldots & \ldots & \ldots \\
0 & 0 & \ldots & I
\end{array}\right)
$$

gives the distinguished vector $\left(\left(\begin{array}{ll}0 & 1 \\ 0 & 0\end{array}\right), 0, \ldots, 0\right)$.

Case 3: $\mathcal{I}_{\boldsymbol{X}}=\mathcal{I}_{1}(b: 1)$ for some $b \in F$. Hence we may assume $\boldsymbol{X}=$ $\left(X_{0}, w_{1} X_{0}, \ldots, w_{n} X_{0}\right)$ with $X_{0}=\left(\begin{array}{ll}0 & b \\ 0 & 1\end{array}\right)$ and $w_{1}, \ldots, w_{n} \in F$. Multiplying $\boldsymbol{X}$ by the invertible matrix

$$
\left(\begin{array}{cccc}
I & -w_{1} I & \ldots & -w_{n} I \\
0 & I & \ldots & 0 \\
\ldots \ldots & \ldots & \ldots & \ldots \\
0 & 0 & \ldots & I
\end{array}\right)
$$

gives the distinguished vector $\left(\left(\begin{array}{ll}0 & b \\ 0 & 1\end{array}\right), 0, \ldots, 0\right)$. The scalar $b$ which appears in this vector depends only on the right ideal $\mathcal{I}_{1}(b: 1)$. 
Case 4: $\mathcal{I}_{\boldsymbol{X}}=\mathcal{I}_{1}$, whence at least one coordinate of $\boldsymbol{X}$ has to be off the Jacobson radical. So the coordinates of $\boldsymbol{X}$ read $X_{i}=\left(\begin{array}{cc}0 & y_{i} \\ 0 & z_{i}\end{array}\right)$ with $z_{0}=1$, say. We introduce the shorthand

$$
d_{i}:=\operatorname{det}\left(\begin{array}{cc}
y_{0} & y_{i} \\
z_{0} & z_{i}
\end{array}\right)=y_{0} z_{i}-y_{i} \text { for } i=1,2, \ldots, n
$$

and proceed in two steps as follows: Define

$$
\boldsymbol{X} \cdot\left(\begin{array}{cccc}
I & -z_{1} I & \ldots & -z_{n} I \\
0 & I & \ldots & 0 \\
\ldots \ldots \ldots \ldots \ldots & \ldots \ldots \\
0 & 0 & \ldots & I
\end{array}\right)=\left(\left(\begin{array}{cc}
0 & y_{0} \\
0 & 1
\end{array}\right),\left(\begin{array}{cc}
0 & -d_{1} \\
0 & 0
\end{array}\right), \ldots,\left(\begin{array}{cc}
0 & -d_{n} \\
0 & 0
\end{array}\right)\right)=: \boldsymbol{X}^{\prime}
$$

Since $\mathcal{I}_{\boldsymbol{X}} \neq \mathcal{I}_{1}\left(y_{0}: 1\right)$, at least one of $d_{1}, d_{2}, \ldots, d_{n}$, say $d_{1}$, is unequal to 0 . Now multiplying $\boldsymbol{X}^{\prime}$ by the invertible matrix

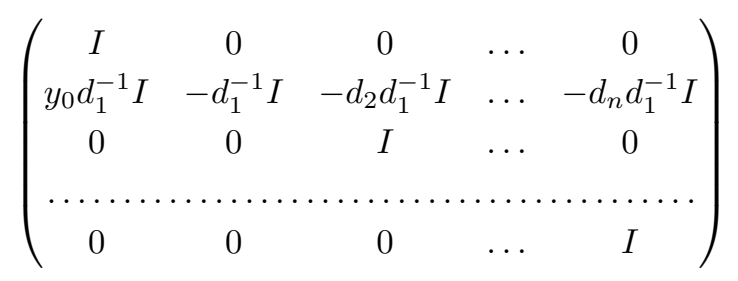

gives the distinguished vector $\left(\left(\begin{array}{ll}0 & 0 \\ 0 & 1\end{array}\right),\left(\begin{array}{ll}0 & 1 \\ 0 & 0\end{array}\right), 0, \ldots, 0\right)$.

Case 5: $\mathcal{I}_{\boldsymbol{X}}=\mathcal{I}_{2}$. We may assume that $X_{i}=\left(\begin{array}{cc}x_{i} & y_{i} \\ 0 & 0\end{array}\right)$ and $x_{0}=1$, because at least one coordinate of $\boldsymbol{X}$ has to be off the Jacobson radical. Multiplying $\boldsymbol{X}$ by the invertible matrix

$$
\left(\begin{array}{cccc}
A_{00} & -X_{1} & \ldots & -X_{n} \\
0 & I & \ldots & 0 \\
\ldots \ldots & \ldots & \ldots & \ldots \\
0 & 0 & \ldots & I
\end{array}\right), \text { where } A_{00}:=\left(\begin{array}{cc}
1 & -y_{0} \\
0 & 1
\end{array}\right)
$$

gives the distinguished vector $\left(\left(\begin{array}{ll}1 & 0 \\ 0 & 0\end{array}\right), 0,0, \ldots, 0\right)$.

Case 6: $\mathcal{I}_{\boldsymbol{X}}=R$. Assume, first, that none of the entries of $\boldsymbol{X}$ is invertible. Hence we have, for example, $X_{0} \in \mathcal{I}_{2} \backslash \mathcal{I}_{1}$ and $X_{1} \in \mathcal{I}_{1} \backslash \mathcal{I}_{2}$. Define

$$
\boldsymbol{X} \cdot\left(\begin{array}{ccccc}
I & 0 & 0 & \ldots & 0 \\
I & I & 0 & \ldots & 0 \\
0 & 0 & I & \ldots & 0 \\
\ldots & \ldots & \ldots & 0 \\
0 & 0 & 0 & \ldots & I
\end{array}\right)=: \boldsymbol{X}^{\prime}
$$

Then $X_{0}^{\prime}=X_{0}+X_{1}$ is a unit. Thus, we may restrict ourselves to the case when one of the entries of $\boldsymbol{X}$, say $X_{0}$, is a unit. Now, multiplying $\boldsymbol{X}$ by the invertible matrix

$$
\left(\begin{array}{ccccc}
X_{0}^{-1} & -X_{0}^{-1} X_{1} & -X_{0}^{-1} X_{2} & \ldots & -X_{0}^{-1} X_{n} \\
0 & I & 0 & \ldots & 0 \\
0 & 0 & I & \ldots & 0 \\
\ldots \ldots \ldots \ldots \ldots \ldots \ldots \ldots \ldots \ldots \ldots \ldots \ldots \ldots & \ldots \ldots \ldots \ldots
\end{array}\right)
$$

gives the distinguished vector $(I, 0, \ldots, 0)$. 
The previous proof shows that for $n \geq 1$ the vectors of $R^{n+1}$ fall into $5+|F|$ orbits.

Lemma 2. Under the action of the general linear group $\mathrm{GL}_{n+1}(R), n \geq 1$, the cyclic submodules of $R^{n+1}$ fall into six orbits with the following representatives:

$$
\begin{aligned}
R(0,0, \ldots, 0) & =\{(0,0, \ldots, 0)\} . \\
R\left(\left(\begin{array}{ll}
0 & 1 \\
0 & 0
\end{array}\right), 0, \ldots, 0\right) & =\left\{\left(\left(\begin{array}{ll}
0 & y \\
0 & 0
\end{array}\right), 0, \ldots, 0\right) \mid y \in F\right\} . \\
R\left(\left(\begin{array}{ll}
0 & 0 \\
0 & 1
\end{array}\right), 0, \ldots, 0\right) & =\left\{\left(\left(\begin{array}{ll}
0 & y \\
0 & z
\end{array}\right), 0, \ldots, 0\right) \mid y, z \in F\right\} . \\
R\left(\left(\begin{array}{ll}
0 & 0 \\
0 & 1
\end{array}\right),\left(\begin{array}{ll}
0 & 1 \\
0 & 0
\end{array}\right), 0, \ldots, 0\right) & =\left\{\left(\left(\begin{array}{ll}
0 & y \\
0 & z
\end{array}\right),\left(\begin{array}{ll}
0 & x \\
0 & 0
\end{array}\right), 0, \ldots, 0\right) \mid x, y, z \in F\right\} . \\
R\left(\left(\begin{array}{ll}
1 & 0 \\
0 & 0
\end{array}\right), 0, \ldots, 0\right) & =\left\{\left(\left(\begin{array}{ll}
x & 0 \\
0 & 0
\end{array}\right), 0, \ldots, 0\right) \mid x \in F\right\} . \\
R(I, 0, \ldots, 0) & =\left\{\left(\left(\begin{array}{ll}
x & y \\
0 & z
\end{array}\right), 0, \ldots, 0\right) \mid x, y, z \in F\right\} .
\end{aligned}
$$

Proof. The assertion follows immediately from the classification of vectors in Lemma 1.

It is worth noting that the cyclic submodule given in (7) is generated by any vector $\boldsymbol{X}$ with $\mathcal{I}_{\boldsymbol{X}}=\mathcal{I}_{1}(b: 1)$ for an arbitrary $b \in F$. This illustrates once more that the classification of cyclic submodules is coarser than the classification of vectors.

In the terminology of $\left[2\right.$, p. 1129] the cyclic ${ }^{2}$ submodules of $R^{n+1}$ are the points of the projective lattice geometry given by $R^{n+1}$. The only free points, i. e., free cyclic submodules, appearing in Lemma 2 are given in (8) and (10). The point in (10) is unimodular, because there exists an $R$-linear form $R^{n+1} \rightarrow R$ which takes $(I, 0, \ldots, 0)$ to $I \in R$. The point in (8) is not unimodular, since none of its vectors is mapped to $I \in R$ under an $R$-linear form. We shall not be concerned with the orbits of the remaining points from Lemma 2 , since none of them is free.

The orbit of (10) under the action of $\mathrm{GL}_{n+1}(R)$ is thus the set of "ordinary" (i. e., unimodular free) points. Note that only the elements of this set are called "points" in [3], [4], and [10]. See also the section on Barbilian spaces in projective lattice geometries in [2, p. 1135-1136]. The orbit of (8) gives rise to the set of "extraordinary" (i. e., non-unimodular free) points of the projective lattice geometry associated with $R^{n+1}$. It is the latter set we shall consider in the sequel, due to its link with the $n$-dimensional projective space $\operatorname{PG}(n, F)$.

\section{Linking non-unimodular free cyclic submod- ules with lines of $\mathrm{PG}(n, F)$}

The free $R$-left module $R^{n+1}$ turns into a $3(n+1)$-dimensional vector space over $F$ by restricting the ring of scalars from $R$ to $F$. (We tacitly do not distinguish between $x \in F$ and the ternion $x \cdot I \in R$.) Each $R$-submodule of $R^{n+1}$ is at the same time an $F$-subspace of this vector space. In this section we focus our

\footnotetext{
${ }^{2}$ In [2] such submodules are called 1-generated rather than "cyclic". The latter term has a different meaning there $[2$, p. 1121].
} 
attention to the $R$-submodule $(\operatorname{rad} R)^{n+1} \subset R^{n+1}$. There exists an obvious $F$-linear bijection between $(\operatorname{rad} R)^{n+1} \rightarrow F^{n+1}$ which is given by

$$
\left(\left(\begin{array}{cc}
0 & y_{0} \\
0 & 0
\end{array}\right),\left(\begin{array}{cc}
0 & y_{1} \\
0 & 0
\end{array}\right), \ldots\left(\begin{array}{cc}
0 & y_{n} \\
0 & 0
\end{array}\right)\right) \mapsto\left(y_{0}, y_{1}, \ldots, y_{n}\right) .
$$

In the proof of the following result we use this mapping to consider $(\operatorname{rad} R)^{n+1}$ as an underlying vector space for the projective space $\operatorname{PG}(n, F)$. Note that we cannot take $\operatorname{rad} R$ (together with addition and multiplication from $R$ ) as the field of scalars for this vector space, but we have to let $F$ play this role.

Theorem 1. Let $R$ be the ring of ternions over a field $F$. The lines of the projective space $\mathrm{PG}(n, F), n \geq 1$, are precisely the intersections of $(\operatorname{rad} R)^{n+1}$ with the non-unimodular free cyclic submodules of the module $R^{n+1}$.

Proof. Under the action of $\mathrm{GL}_{n+1}(R)$ on $R^{n+1}$ the set $(\operatorname{rad} R)^{n+1}$ is invariant. Consequently, $\mathrm{GL}_{n+1}(R)$ acts as an $F$-linear transformation on $(\operatorname{rad} R)^{n+1}$. Conversely, given an $F$-linear bijection of $(\operatorname{rad} R)^{n+1}$ it will correspond, in terms of the coordinates given by (11), to a unique matrix $\left(a_{i j}\right) \in \mathrm{GL}_{n+1}(F)$. This matrix over $F$ determines the matrix $\left(a_{i j} I\right) \in \mathrm{GL}_{n+1}(R)$ which in turn induces the given transformation on $(\operatorname{rad} R)^{n+1}$. Thus the $F$-linear bijections of $(\operatorname{rad} R)^{n+1}$ are precisely the $R$-linear bijections of $R^{n+1}$ restricted to $(\operatorname{rad} R)^{n+1}$. We add in passing that this action of $\mathrm{GL}_{n+1}(R)$ on $(\operatorname{rad} R)^{n+1}$ is not faithful.

The non-unimodular free cyclic submodule $(8)$ meets $(\operatorname{rad} R)^{n+1}$ in a twodimensional $F$-subspace or, said differently, in a line of $\operatorname{PG}(n, F)$. All nonunimodular free cyclic submodules of $R^{n+1}$ (and, likewise, all lines of PG $(n, F)$ ) form an orbit under the action of the group $\mathrm{GL}_{n+1}(R)$. This proves the assertion.

The previous result describes only the lines of $\mathrm{PG}(n, F)$ as certain subsets of $(\operatorname{rad} R)^{n+1}$. However, for $n \geq 2$ this implies that also the points of this projective space are known: A subset $p$ of $(\operatorname{rad} R)^{n+1}$ is a point if, and only if, there exist non-unimodular free cyclic submodules $R \boldsymbol{X}$ and $R \boldsymbol{Y}$ of $R^{n+1}$ such that

$$
\begin{gathered}
R \boldsymbol{X} \cap(\operatorname{rad} R)^{n+1} \neq R \boldsymbol{Y} \cap(\operatorname{rad} R)^{n+1}, \\
p=R \boldsymbol{X} \cap R \boldsymbol{Y} \cap(\operatorname{rad} R)^{n+1}, \text { and }|p|>1 .
\end{gathered}
$$

Thus the vectors of $(\operatorname{rad} R)^{n+1}$ together with the "traces" of the non-unimodular free cyclic submodules of $R^{n+1}$ completely determine the structure of $\mathrm{PG}(n, F)$ for $n \geq 2$. There is yet another approach to points which makes use of unimodular free cyclic submodules. It works even for $n \geq 1$ : The points of $\operatorname{PG}(n, F)$ are precisely the intersections of $(\operatorname{rad} R)^{n+1}$ with the unimodular free cyclic submodules of the module $R^{n+1}$. This follows like in the proof of Theorem 1 from the fact that the meet of the submodule (10) with $(\operatorname{rad} R)^{n+1}$ is a point of $\mathrm{PG}(n, F)$, and from the actions of $\mathrm{GL}_{n+1}(R)$ on $R^{n+1}$ and $(\operatorname{rad} R)^{n+1}$.

Finally, we note that Theorem 1 also furnishes the proof of the validity of the conjecture raised in [8] about the connection between non-unimodular free cyclic submodules of $R^{3}$, where $R$ is the ring of ternions over a Galois field $\mathrm{GF}(q)$, and lines of the projective plane $\mathrm{PG}(2, q)$. 


\section{Combinatorics of the finite case}

We assume throughout this section that $F$ is a Galois field $\operatorname{GF}(q)$ with $q$ elements. So $|R|=q^{3}$, and the number of units in $R$ is given by $\left|R^{*}\right|=q(q-1)^{2}$.

Our first aim is to count the numbers $m_{i}$ of vectors of $R^{n+1}$ fitting into cases $i=1,2, \ldots, 6$ according to the classification from the previous section. All of these numbers are non-zero due to our general assumption $n \geq 1$, which is also adopted in this section.

Case 1: The zero-vector is the only vector arising in this case, whence $m_{1}=1$.

Case 2: There are $|\operatorname{rad} R|^{n+1}=q^{n+1}$ vectors with entries from the radical of $R$, including the zero-vector. We obtain therefore $m_{2}=q^{n+1}-1$.

Case 3: First we consider a fixed $b \in F$. Any vector $\boldsymbol{X}$ with $\mathcal{I}_{\boldsymbol{X}}=\mathcal{I}_{1}(b: 1)$ has the form

$$
\left(w_{0}\left(\begin{array}{ll}
0 & b \\
0 & 1
\end{array}\right), w_{1}\left(\begin{array}{ll}
0 & b \\
0 & 1
\end{array}\right), \ldots, w_{n}\left(\begin{array}{ll}
0 & b \\
0 & 1
\end{array}\right)\right)
$$

with $(0,0, \ldots, 0) \neq\left(w_{0}, w_{1}, \ldots, w_{n}\right) \in F^{n+1}$. As $b$ varies in $F$, we get $m_{3}=$ $q\left(q^{n+1}-1\right)$.

Case 4: There are $\left|\mathcal{I}_{1}\right|^{n+1}=q^{2(n+1)}$ vectors with entries from the ideal $\mathcal{I}_{1}$, including the vectors from cases 1,2 , and 3 . Hence

$$
m_{4}=q^{2(n+1)}-m_{3}-m_{2}-m_{1}=q\left(q^{n}-1\right)\left(q^{n+1}-1\right) .
$$

Case 5: We proceed as before and obtain from $\left|\mathcal{I}_{2}\right|=q^{2}$ that

$$
m_{5}=q^{2(n+1)}-m_{2}-m_{1}=q^{n+1}\left(q^{n+1}-1\right) .
$$

Case 6: All remaining vectors fall into this case. We read off from $\left|R^{n+1}\right|=$ $q^{3(n+1)}$ that

$$
m_{6}=\left|R^{n+1}\right|-m_{5}-m_{4}-m_{3}-m_{2}-m_{1}=q^{n+1}\left(q^{n+1}-1\right)^{2} .
$$

We consider now the set $\mathcal{N}$ of all vectors of $R^{n+1}$ which belong to at least one non-unimodular free cyclic submodule of $R^{n+1}$. For this set to be non-empty we must have $n \geq 1$. Under these circumstances the set $\mathcal{N}$ comprises precisely the $q^{2(n+1)}$ vectors fitting into cases $1-4$ according to our classification.

Theorem 2. Let $R$ be the ring of ternions over $\mathrm{GF}(q)$. There are precisely

$$
\mu:=\frac{\left(q^{n}-1\right)\left(q^{n+1}-1\right)}{(q-1)^{2}}
$$

non-unimodular free cyclic submodules in $R^{n+1}$ for $n \geq 1$. The number of such submodules containing a vector $\boldsymbol{X} \in R^{n+1}$ equals

$$
\begin{aligned}
\mu_{1}:=\mu & \text { if } & \mathcal{I}_{\boldsymbol{X}}=0, \\
\mu_{2}:=\frac{(q+1)\left(q^{n}-1\right)}{q-1} \quad \text { if } & & \mathcal{I}_{\boldsymbol{X}}=\operatorname{rad} R, \\
\mu_{3}:=\frac{q^{n}-1}{q-1} & \text { if } & \mathcal{I}_{\boldsymbol{X}}=\mathcal{I}_{1}(b: 1) \text { for some } b \in F, \\
\mu_{4}:=1 & \text { if } & \mathcal{I}_{\boldsymbol{X}}=\mathcal{I}_{1} .
\end{aligned}
$$


Proof. Equation (16) holds trivially. The number of non-unimodular free cyclic submodules is $m_{4} /\left|R^{*}\right|$ which yields (12) and (13). In order to establish (14) we count in two ways the number of pairs $(\boldsymbol{X}, R \boldsymbol{Y})$, where $R \boldsymbol{Y}$ is a non-unimodular free cyclic submodule and the vector $\boldsymbol{X} \in R \boldsymbol{Y}$ is subject to $\mathcal{I}_{\boldsymbol{X}}=\operatorname{rad} R$.

First we fix $\boldsymbol{Y}$ and count the number of appropriate vectors $\boldsymbol{X}$. Since all vectors $\boldsymbol{Y}$ with $\mathcal{I}_{\boldsymbol{Y}}=\mathcal{I}_{1}$ are in one orbit of $\mathrm{GL}_{n+1}(R)$, it is sufficient to consider as $\boldsymbol{Y}$ the distinguished vector obtained in case 4 . By (8), the vectors of $R \boldsymbol{Y}$ have the form

$$
\left(\left(\begin{array}{ll}
0 & y \\
0 & z
\end{array}\right),\left(\begin{array}{ll}
0 & x \\
0 & 0
\end{array}\right), 0, \ldots, 0\right) \text { with } x, y, z \in F .
$$

Such a vector has the required properties if, and only if, $z=0$ and $(x, y) \neq(0,0)$. Hence there are $q^{2}-1$ vectors of this kind. Consequently, as $\boldsymbol{Y}$ varies also, the number of pairs $(\boldsymbol{X}, R \boldsymbol{Y})$ is equal to

$$
\left(q^{2}-1\right) \cdot \mu=\frac{(q+1)\left(q^{n}-1\right)\left(q^{n+1}-1\right)}{(q-1)} .
$$

Counting in a different way, we find that this number equals $\mu_{2} m_{2}$, whence indeed

$$
\mu_{2}=\frac{(q+1)\left(q^{n}-1\right)}{(q-1)} .
$$

The proof of (15) can be accomplished in the same fashion. The coordinates of a vector given in (17) generate a right ideal $\mathcal{I}_{1}(b: 1)$ for some $b \in F$ if, and only if, $z \neq 0$ and $x=0$. The last condition is due to the fact that second ternion coordinate of the vector appearing in (17) has to be a scalar multiple of the first one. Hence there are $q(q-1)$ vectors of this kind. This gives

$$
q(q-1) \cdot \mu=\frac{q\left(q^{n}-1\right)\left(q^{n+1}-1\right)}{(q-1)}=\mu_{3} m_{3}=\mu_{3} \cdot q\left(q^{n+1}-1\right)
$$

from which the formula for $\mu_{3}$ is immediate.

We notice that

$$
\begin{aligned}
\mu=\mu_{1} & =|\mathrm{PG}(n-1, q)| \cdot \mid \mathrm{PG}(n, q)) \mid, \\
\mu_{2} & =|\mathrm{PG}(n-1, q)| \cdot|\mathrm{PG}(1, q)|, \\
\mu_{3} & =|\mathrm{PG}(n-1, q)| .
\end{aligned}
$$

Thus for $n \geq 2$ these numbers are distinct and all of them are greater than $\mu_{4}=1$. Under these circumstances the four types of vectors in $\mathcal{N}$ can be distinguished by the number of non-unimodular free cyclic submodules in which they are contained. Consequently, the set $\mathcal{N}$ and the family of non-unimodular free cyclic submodules determine the lines and points of the projective space $\mathrm{PG}(n, q)$ according to Theorem 1 and the subsequent remarks. This provides now the theoretical background for the Fano-Snowflake from [8], which is depicted in Figure 1, and puts the construction from there in a general context by allowing an arbitrary dimension $n \geq 2$ and any prime power $q$. 


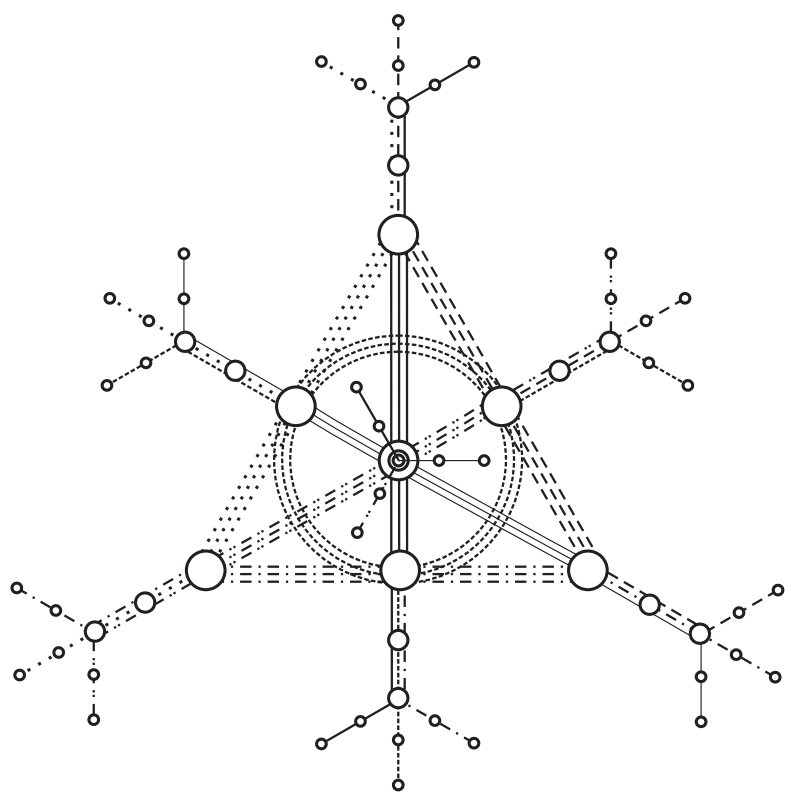

Figure 1: The "Fano-Snowflake" — a diagrammatic illustration of the $n=q=2$ case. The $2^{6}-1=63$ vectors of $\mathcal{N} \backslash\{(0,0,0)\}$ are represented by circles, whose sizes reflect the number of non-unimodular free cyclic submodules of $R^{3}$ they are contained in. As the zero vector is not shown, each submodule of this kind is represented by $2^{3}-1=7$ circles lying on a common polygon; three big, two medium-sized, and two small circles corresponding, respectively, to the vectors from case $2\left(\mu_{2}=9\right)$, case $3\left(\mu_{3}=3\right)$, and case $4\left(\mu_{4}=1\right)$. The patterns of the $\mu=21$ polygons were chosen in such a way to make (the lines of) the Fano plane PG(2,2) sitting in the middle of the "snowflake" readily discernible. The illustration is essentially three-dimensional. There is also a single "vertical branch" of the "snowflake" which emanates from the the middle of the figure and ramifies into three smaller branches.

\section{Conclusion}

Given the free left $R$-module $R^{n+1}, n \geq 1$, of an arbitrary ring of ternions $R$, we provide a complete classification of the vectors from $R^{n+1}$ (Lemma 1) and the cyclic submodules generated by them (Lemma 2), up to the action of the group $\mathrm{GL}_{n+1}(R)$. There exist altogether $5+|F|$ distinct orbits of vectors and six (two free, one of them non-unimodular) ones of submodules. The non-unimodular free cyclic submodules are linked with the lines of $\mathrm{PG}(n, F)$ (Theorem 1). In the finite case, we count explicitly the total number of non-unimodular free cyclic submodules as well as the cardinalities of their subsets passing through a given vector (Theorem 2). In light of the fact that there are only few papers on projective geometries over ternions, we hope that our findings will stir the interest of mathematicians into a more systematic treatment of exciting open problems in this particular branch of ring geometries, and will also prove fruitful for envisaged applications of projective geometries over ternions in quantum physics. 


\section{Acknowledgments}

The work was supported by the VEGA grant agency projects Nos. 6070 and 7012, as well as by the Action Austria-Slovakia project No. 58s2. We thank Petr Pracna (Prague) for providing us with the electronic version of the figure.

\section{References}

[1] A. Blunck and A. Herzer, Kettengeometrien. Eine Einführung, Berichte aus der Mathematik, Shaker, Aachen, 2005.

[2] U. Brehm, M. Greferath and S. E. Schmidt, Projective geometry on modular lattices, in Handbook of Incidence Geometry, F. Buekenhout, ed., Elsevier, Amsterdam, 1995, pp. 1115-1142.

[3] J. Depunt, Sur la géométrie ternionienne dans le plan, Bull. Soc. Math. Belg. 11 (1959) 123-133.

[4] J. Depunt, Grondslagen van de analytische projectieve ternionenmeetkunde van het platte vlak, Verh. Konink. Acad. Wetensch. Lett. Schone Kunst. België, Kl. Wetensch. 22 (63) (1960) 99 pp.

[5] H. Havlicek and M. Saniga, Projective ring line of an arbitrary single qudit, J. Phys. A: Math. Theor. 41 (2008) 015302.

[6] M. Planat and A.-C. Baboin, Qudits of composite dimension, mutually unbiased bases and projective ring geometry, J. Phys. A: Math. Theor. 40 (2007) F1005-F1012.

[7] M. Saniga, A fine structure of finite projective ring lines. An invited talk given at the workshop on Prolegomena for Quantum Computing, held in Besançon (France), November 21-22, 2007; viewgraphs of the talk available on-line at http://hal.archives-ouvertes.fr/hal-00199008.

[8] M. Saniga, H. Havlicek, M. Planat and P. Pracna, Twin "Fanosnowflakes" over the smallest ring of ternions, SIGMA 4 (2008) Paper 050; arXiv:0803.4436.

[9] M. Saniga, M. Planat, M. R. Kibler and P. Pracna, A classification of the projective lines over small rings, Chaos, Solitons and Fractals 33 (2007) 1095-1102.

[10] F. D. Veldkamp, Geometry over rings, in Handbook of Incidence Geometry, F. Buekenhout, ed., Elsevier, Amsterdam, 1995, pp. 1033-1084.

Hans Havlicek

Institut für Diskrete Mathematik und Geometrie, Technische Universität

Wiedner Hauptstraße 8-10/104, A-1040 Wien, Austria

email: havlicek@geometrie.tuwien.ac.at

Metod Saniga

Astronomical Institute, Slovak Academy of Sciences

SK-05960 Tatranská Lomnica, Slovak Republic

email: msaniga@astro.sk 